\title{
Absceso hepático como presentación inicial de la enfermedad invasora por Streptococcus pneumoniae
}

\author{
Leonardo Gilardi y María Eugenia Dellepiane
}

\section{Hepatic abscess as first manifestation of pneumococcal invasive disease}

Pneumococcal invasive disease is an important cause of morbidity and mortality in different population groups. Most cases originate from an airway infection. We describe a patient with diabetes mellitus who presented a liver abscess as first manifestation of pneumococcal invasive disease, without respiratory symptoms. The patient was treated with percutaneous drainage and systemic antibiotics with good results. Streptococcus pneumoniae should be considered among the possible etiologies of hepatic abscess, even in absence of respiratory symptoms.

Key words: Streptococcus pneumoniae, pneumococcal invasive disease, liver abscess.

Palabras clave: Streptococcus pneumoniae, enfermedad invasiva por neumococo, absceso hepático.
Ciudad Autónoma de Buenos Aires. Argentina Osecac

Programa de Guías de Práctica Clínica (LG)

Servicio de Internación (MED)

Conflicto de intereses: ninguno. Patrocinio: ninguno.

Recibido: 25 de octubre de 2010 Aceptado: 31 de marzo de 2011

Correspondencia a:

Leonardo Gilardi

revision_guias@yahoo.com.ar

\section{Introducción}

\section{L} a enfermedad invasora por Streptococcus pneumoniae es una causa importante de morbi-mortalidad en diferentes grupos poblacionales. La mayor parte de los casos se origina a partir de un foco infeccioso primario de origen respiratorio ${ }^{1}$. Sin embargo, no se ha descrito con anterioridad al absceso hepático como forma de presentación inicial de esta afección.

\section{Observación clínica}

Presentamos un enfermo de 60 años, con antecedentes de diabetes mellitus tipo 2 con inadecuado control metabólico, quien se presentó a la consulta por dolor abdominal continuo localizado en el hipocondrio derecho, asociado con la presencia de hepatomegalia en el examen físico. El paciente negaba la presencia de tos o de disnea. La semiología torácica y la radiografía simple de tórax eran normales. Mediante una tomografía computarizada se reconocieron sendas formaciones hipodensas en el lóbulo hepático derecho, de aspecto irregular, la mayor de las cuales medía $131 \mathrm{~mm}$ de diámetro mayor. En el mismo estudio se observó una mínima efusión pleural, bibasal, e infiltrados compatibles con la presencia de atelectasias. Se llevó a cabo un drenaje por vía percutánea, con la obtención de material purulento con cultivo positivo para $S$. pneumoniae sensible a penicilina (CIM $0,1 \mu \mathrm{g} / \mathrm{ml}$ ). Los hemocultivos fueron negativos. Se realizó además un ecocardiograma en el cual no se reconocieron vegetaciones valvulares o endocárdicas. La administración de aminopenicilinas intravenosas durante los primeros siete días se asoció con evolución favorable, para continuar posteriormente el tratamiento con amoxicilina por vía oral, con la que enteró seis semanas.

\section{Discusión y conclusión}

Se destaca que los sujetos con diabetes mellitus tipo 2 se caracterizan por una mayor predisposición a la enfermedad invasora neumocóccica. ${ }^{1}$ Sin embargo, el absceso hepático no constituye una forma frecuente de presentación de esta afección, en especial en ausencia de hemocultivos positivos. En cambio, hasta $25 \%$ de los abscesos hepáticos se caracterizan por la presencia de bacteriemia, siendo Staphylococcus aureus el microrga- nismo predominante ${ }^{2}$. La tomografía computarizada constituye un método diagnóstico adecuado, si bien la mayor parte de las lesiones resultan menores que las verificadas en nuestro paciente ${ }^{3}$.

El tratamiento empleado con mayor frecuencia consiste en la combinación de procedimientos de radiología intervencional con antimicrobianos sistémicos, en coincidencia con la estrategia implementada en este caso ${ }^{2}$.

De esta manera, sugerimos que S.pneumoniae debería considerarse entre las etiologías posibles de los abscesos hepáticos en sujetos con factores de riesgo para enfermedad invasora por este agente, aún en ausencia de manifestaciones respiratorias o de hemocultivos positivos.

\section{Resumen}

La enfermedad invasora por Streptococcus pneumoniae es una causa importante de morbi-mortalidad en diferentes grupos poblacionales. La mayor parte de los casos se origina a partir de un foco infeccioso primario de origen respiratorio. Se describe el caso de un paciente diabético en el cual esta grave enfermedad se presentó de manera inicial como un absceso hepático sin manifestaciones respiratorias asociadas. El enfermo respondió de manera apropiada al tratamiento quirúrgico percutáneo y a la administración de antimicrobianos sistémicos. Se propone la consideración de $S$. pneumoniae entre las etiologías posibles de los abscesos hepáticos en sujetos con factores de riesgo para enfermedad invasora por este microorganismo, aún en ausencia de síntomas respiratorios.

\section{Referencias}

1.- Musher D M. Streptococcus pneumoniae. In: Mandell, Douglas, and Bennett's Principles \& Practice of Infectious Diseases. Mandell GL; Bennett J. \& Dolin R eds. $7^{\text {th }}$ ed. Philadelphia: Churchill Livingstone, Elsevier; 2010. p. 2633-41.

2.- Fantuzzi A, Albertz N, Valenzuela A, Estuardo N, Castro A. Absceso hepático: Serie de 107 casos y revisión de la literatura. Rev Chil Infectol 2009; 26 (1): 49-53.

3.- Wang C L, Guo X J, Qiu S B, Lei Y, Yuan Z D, Dong H B, et al. Diagnosis of bacterial hepatic abscess by CT. Hepatobiliary Pancreat Dis Int 2007; 6: 271-5. 